\title{
Selection of aptamers to Neisseria meningitidis and Streptococcus pneumoniae surface specific proteins and affinity assay using thin film AlN resonators
}

\author{
José M. Escolano , Bárbara Díaz-Durán , Mario DeMiguel-Ramos , Jimena Olivares , \\ Morten A. Geday , Enrique Iborra
}

Keywords:

Meningit is

SOMAmer

AlN gravimetric biosensor

PavA

FHbp

\begin{abstract}
A B S T R A C T
The first steps in the development of an aptasensor for bacterial meningitis diagnosis in real time are described: slow off-rate modified aptamers (SOMAmer) selection, gravimetric sensor fabrication and functionalization of its active surface. SOMAmers polyclonal populations were generated to surface specific proteins PavA (S. pneumoniae) and FHbp (N. meningitidis) by systematic evolution of ligands by exponential enrichment method (SELEX). After eight rounds, they were tested by direct enzyme-linked oligonucleotide assays (ELONA) and by high frequency $(1.4 \mathrm{GHz})$ gravimetric sensors based on thin film AlN resonators operating in shear mode. The sensing surface of the resonators was functionalized using a silane-glutaraldehyde based protocol and the binding of PavA and FHbp was measured in real time. The SOMAmers polyclonal populations showing the best ELONA results have been also tested using gravimetric sensors and the binding to the protein functionalized surface was measured in real time showing positive results. These first steps towards the development of an aptasensor for the targeted bacteria demonstrate the potential of the method for sensitive, rapid, and cost effective detection of bacterial meningitis.
\end{abstract}

\section{Introduction}

Bacterial meningitis is one of the ten leading causes of death due to infectious diseases worldwide. About $10 \%$ of the patients die within 24-48 h of the onset of symptoms and long-term neurological sequelae occurs in $10-20 \%$ of survivors [1]. Moreover, bacterial meningitis spreads rapidly among people through coughing, sneezing or close contact [2]. Therefore, a quickly and highly sensitive method of diagnosis is essential for early treatment of the patients using the appropriate therapeutic approach and to prevent the outbreak $[3,4]$.

Neisseria meningitidis and Streptococcus pneumoniae are most commonly associated with bacterial meningitis [4]. However their early clinical diagnosis are usually nonspecific and rapid identification of bacteria can be critical to successful treatment [5]. Definitive diagnosis is usually established by conventional methods of bacterial detection as culture or immunological tests of bacterial antigens, which take longer than the therapeutic window [6]. New molecular techniques as real-time polymerase chain reaction (PCR) analysis can be completed faster, within several hours, but still require specialized personnel, equipment and reagents and are consequently costly [7]. Therefore, there is an urgent demand for faster, cost effective and sensitive Point of Care (PoC) tests of bacteria causing meningitis that do not require multistep processing and purification. Biosensors could be the best alternative to address this issue [8].

Regarding bacterial meningitis sensing, two classes of biosensors have been developed. The first type requires bacterial disruption or lysis in order to liberate a specific component of the targeted bacteria. The second type do not require any preprocessing and it targets whole bacteria or bacterial antigens. Biosensors in the first category detect meningococcus, pneumococcus and other bacteria components such as DNA and proteins by different methods like fluorescence [9], surface plasmon resonance (SPR) [10], quartz crystal microbalance (QCM) [11], piezoelectric [12], electroluminescence [13], electro-chemical [14,15] or by matrix-assisted laser desorption/ionization time-of-flight mass spectrometry (MALDI- 
MS) [16-18]. The major disadvantage of these systems is the requirement of sample processing and the use of extra reagents, which need well trained personnel. More direct diagnosis have been reached with whole bacteria detection by using electrochemical sensors [19] or meningococcal antigens with QCM [20], but these systems require the use of labeling reagents for the chemical reaction in the first case and for increasing sensitivity in the second. Label-free biosensors for direct detection of whole bacteria or bacterial antigens are much more desirable for rapid and cost-effective PoC sensors.

In recent years, aptamers have emerged as an excellent alternative to antibodies as bio-recognition elements in affinity biosensors [21] because of their chemical stability and ease to be modified. SOMAmers are an improved evolution of standard aptamers, which are made from single-stranded DNA (ssDNA) that contains deoxyuridine residues modified at their 5-position with hydrophobic aromatic functional groups that mimic amino acid side chains [22]. These features lead to exceptional affinity and better kinetic properties of these reagents compared to standard RNA or DNA aptamers and they increase the success rate to select high-quality ligands for a wide range of targets. SOMAmers are selected in vitro in the same way as standard aptamers by the systematic evolution of ligands by exponential enrichment (SELEX) method, which consists of multiple cycles of selection, partitioning, and amplification [23]. Advantages of SOMAmers over antibodies include lower molecular weight (low steric hindrance, allowing higher loading density and therefore higher signal intensities), chemical stability to heat, drying and solvents, reversible renaturation, ease of reagent manufacturing, consistent lot-to-lot performance and lower cost.

Gravimetric sensors based on bulk acoustic wave (BAW) resonators work under the principle of the resonant frequency shift due to a mass loading [24]. They have shown significant potential as label-free biosensors [24] due to their high sensitivity, low detection limit, and low cost. Since the sensitivity increases with resonant frequency [25], high frequency resonators are preferred. This has boosted the research in thin film piezoelectric materials, such as AlN or $\mathrm{ZnO}$, for BAW resonators [26,27]. Film bulk acoustic resonators (FBAR) display two different resonance modes: longitudinal and shear. If the FBAR operates in liquid media, it is necessary to excite the shear modes of acoustic propagation, since these modes experience a lower attenuation than longitudinal ones when working in liquids. Among thin film piezoelectric materials, AlN displays some additional advantages for in-liquid operation owing to its high acoustic velocity and its better resistance to liquids. The technology for fabricating AlN based BAW resonators, either as solidly mounted resonators or as free-standing membrane resonators is mature and already in production for telecommunication electronic applications [28]. Therefore, the extension to sensors market should be straightforward.

In this paper we describe the first steps in the development of a high frequency gravimetric aptasensor for detection of Streptococcus pneumoniae and Neisseria meningitidis. This first step consists in the development of SOMAmers to bacteria surface specific proteins, which ensure the detection of the bacteria. The chosen proteins were PavA and FHbp specific from the surface of those bacteria. PavA is a fibronectin-binding protein with $62 \mathrm{kDa}$ of molecular weight and localized at the outer cell surface of Streptococcus pneumoniae which has been identified as a virulence factor [29]. FHbp or factor $\mathrm{H}$ binding protein is a $27 \mathrm{kDa}$ of molecular weight lipoprotein from Neisseria meningitidis, which is important for the survival of the bacteria in human blood and it is a component of two recently licensed vaccines against serogroup B meningococcus (MenB) [30]. After the SOMAmers selection, their specificity and affinity to the targeted proteins were tested by a conventional technique (ELONA) and by high frequency gravimetric sensors functionalized with the proteins.

\section{Materials and methods}

\subsection{Synthesis of recombinant proteins PavA and FHbp}

The protein sequences for recombinant synthesis have been provided by research groups specialized in meningitis-causing bacteria. The FHbp sequence from Neisseria meningitidis has been provided by the Group of Molecular Analyses and Vaccine Development for Meningococcal Meningitis from the University of Santiago de Compostela (Spain) and it corresponds to UniProtKB accession number A1E5L5. The PavA sequence from Streptococcus pneumoniae has been provided by the Research Group "Host-parasite interplay in pneumococcal infection" from the Biological Research Center (CIB-CSIC) (Spain) and it corresponds to the UniProtKB accession number Q9RNF3. Both proteins were synthesized by the biotechnology company Biologics Corp based on the sequences provided by us. The synthesis includes gene synthesis, sub-cloning into expression vectors, scale-up expression, and protein purification. For protein isolation one His6-tag was added to each sequence resulting in $64 \mathrm{kDa}$ molecules for PavA and $30 \mathrm{kDa}$ for FHbp. The synthesis method included SupernateIN ${ }^{\mathrm{TM}}$ protein expression to select an optimal condition in which the protein is expressed in soluble form in vivo and purified as an independent protein (no complex with other proteins). Both proteins have been assessed by SDS-PAGE and western-blot at the provider company and, after receiving them, in our laboratory, ensuring a purity $>90 \%$.

\subsection{SOMAmer selection and synthesis}

SELEX was performed as previously described [22,23], using $1 \mathrm{nMol}\left(10^{14}-10^{15}\right)$ sequences of modified DNA sequences containing 40 consecutive randomized positions flanked by fixed sequences required for PCR amplification. One library including 5-tryptaminocarbonyl-dU (TrpdU) (Jena Bioscience) modification was used in the SELEX experiment. SBIT ( $40 \mathrm{mM}$ HEPES pH 7.5, $125 \mathrm{mM} \mathrm{NaCl}, 5 \mathrm{mM} \mathrm{KCl}, 1 \mathrm{mM} \mathrm{MgCl}_{2}, 1 \mathrm{mM} \mathrm{CaCl} 2$ and $0.05 \%$ Tween-20) buffer was used throughout SELEX and the subsequent binding assays. Eight rounds of selection were carried out for each SELEX experiment. At the beginning of third round, a kinetic challenge with $10 \mathrm{mM}$ dextran sulfate (Sigma-Aldrich) was performed to favor slow off-rates. Partitioning of the aptamer-target complexes was achieved with paramagnetic Talon Dynabeads ${ }^{(8)}$ (Invitrogen) that binds the His6-tag on the recombinant proteins and selected aptamers were amplified using KOD XL DNA polymerase (Novagen EMD Millipore). SOMAmers polyclonal populations obtained after the fifth and eighth rounds of SELEX were compared for affinity and specificity to their targets by ELONA. For ELONA test SOMAmers were modified with digoxigenin at $5^{\prime}$ position. The best of them were incubated against their target on gravimetric sensors.

\subsection{Direct ELONA}

For direct ELONA assays, FHbp or PavA proteins were coated on 96 well microtiter plate Maxisorp (NUNC) by incubation of $0.5 \mu \mathrm{g} /$ well in $100 \mu \mathrm{l}$ SBIT buffer overnight at $4^{\circ} \mathrm{C}$. The plates were washed and blocked with SBIT/1\% BSA. Polyclonal aptamers modified with digoxigenin were added in serial dilutions ( $40 \mathrm{nM}$ to $5 \mathrm{nM}$ ) and allowed to bind for $60 \mathrm{~min}$ at room temperature. After washing, Anti-Digoxigenin-POD, Fab fragments (Roche) were added (1/1000 diluted) in SBIT/BSA $1 \%$ buffer and incubated for $60 \mathrm{~min}$ at room temperature. Aptamers bound were detected with ABTS 

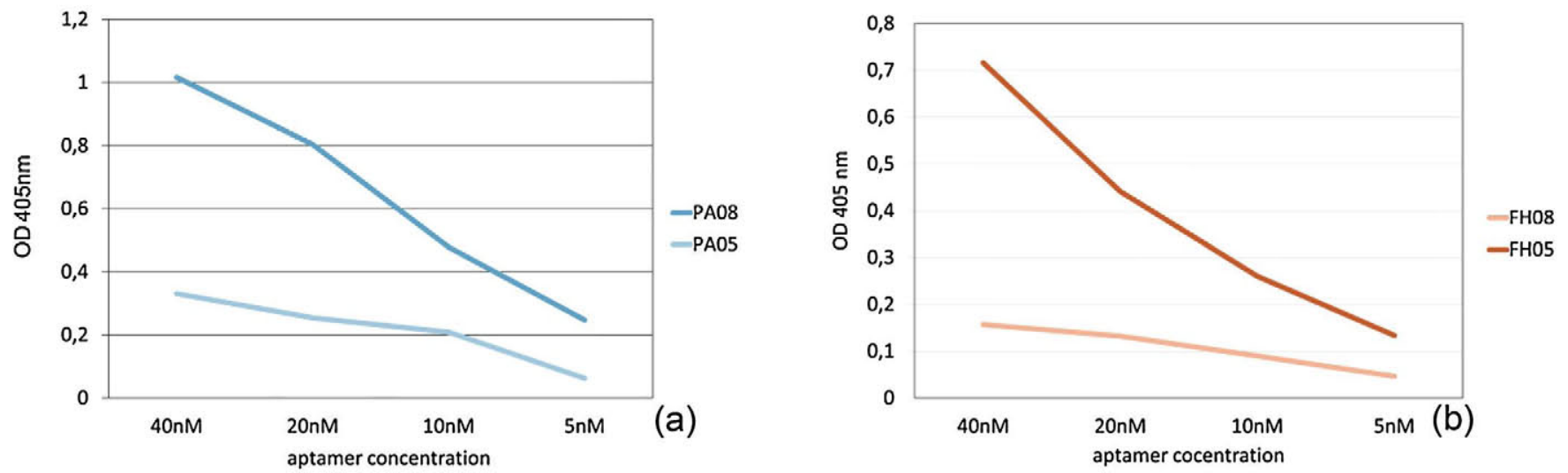

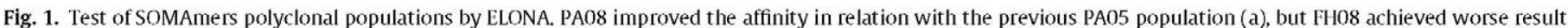
in relation to its previous population FH05 (b).

Chromogenic substrate for peroxidase (Roche) and absorbance at $405 \mathrm{~nm}$ was measured each two minutes.

\subsection{AlN resonator as gravimetric sensor}

The core of the sensing technology is a piezoelectric resonator, which consists in a thin AlN film deposited between two iridium electrodes. This AIN layer was sputtered with its wurtzite microcrystals tilted around $25^{\circ}$ with respect to the normal to allow operation in acoustic shear mode. To achieve the piezoelectric layer with tilted grains a two-stage reactive sputtering process is employed [31]. The thickness of this layer was around $700 \mathrm{~nm}$ and it sets the resonant frequency of the sensor at around $1.4 \mathrm{GHz}$. The acoustic isolation of the piezoelectric stack was achieved using a fully insulating acoustic reflector. On the top electrode a $\mathrm{SiO}_{2}$ thin film (50 nm-thick) was deposited as platform for sensor functionalization. Details of the device fabrication and its use as aptasensor against thrombin can be found in [32]. Assessment with these sensors was performed by monitoring the evolution of frequency shift with time when the different biological species are passing through a fluidic cell where the sensor is attached. The resonant frequency was derived from the maximum of the real part of the electrical admittance measured with a network analyzer. The floor noise obtained in the frequency shift measurements is around $1 \mathrm{kHz}$, which represents less than $1 \mathrm{ppm}$ of the resonant frequency. The mass sensitivity of this type of sensor is around $1800 \mathrm{kHz} / \mathrm{pg} \cdot \mathrm{cm}^{2}$ [32].

\subsection{Assembling PavA and FHbp recombinant proteins onto BAW sensors}

To cover the top of the resonator surface with the recombinant proteins, first it was treated in an oxygen plasma at 13.3 Pa during 2 min to generate $\mathrm{OH}$ groups onto the $\mathrm{SiO}_{2}$ coated top electrode surface. In a second step, the resonator was incubated in a solution of APTES $2 \%$ in absolute ethanol for one hour at room temperature. The surface was washed with absolute ethanol, dried with $\mathrm{N}_{2}$ and cured at $110^{\circ} \mathrm{C}$ in oven during $1 \mathrm{~h}$. After that, the resonator was incubated in glutaraldehyde (GA) $1 \%$ for $1 \mathrm{~h}$ to bind the GA to the silane. We obtained a surface with free aldehyde groups that could bind to primary amine groups $\left(\mathrm{NH}_{2}\right.$-) inside the proteins. The recombinant proteins were incubated at $20 \mu \mathrm{g} / \mathrm{ml}$ in $\mathrm{NaCl} 50 \mathrm{mM}$ for $1 \mathrm{~h}$ at room temperature to finally cover the active surface of the resonator. To avoid nonspecific binding the surface was incubated with $\mathrm{BSA} 1 \%$ in $\mathrm{NaCl} 50 \mathrm{mM}$ for $1 \mathrm{~h}$ at room temperature. Finally, the resonator was immersed in SBIT containing BSA 1\% to equilibrate the surface before the assay.

\section{Results and discussion}

\subsection{Selection of SOMAmers against PavA and FHbp proteins}

PavA and FHbp recombinant proteins were synthesized with His6-tag in order to enable aptamer selection. Previous to the selection the purity was tested by SDS-PAGE and Coomassie Brilliant Blue. First round of SELEX started from one single strand DNA library including dUTP modified at the 5-position: 5-tryptaminocarbonyl-dU (TrpdU). At the beginning of the third round, SELEX conditions were changed each round to favor slow off-rates: $10 \mathrm{mM}$ dextran sulfate was added, binding time was reduced, incubation time of washing was increased and we introduced counter SELEX against different recombinant proteins that had been synthesized using the same system. The SOMAmers polyclonal populations were tested after five and eight rounds of SELEX by direct ELONA (Fig. 1). Four polyclonal populations were obtained, two from each protein: FH05-FH08 from FHbp selection and PA05-PA08 from PavA. The polyclonal populations that achieved the best result were chosen for further analysis with the gravimetric sensor. Such populations were PA08 and FH05. Noteworthy, evolution of affinity in both populations was different. Contrary to what had been expected, FH08 population lost the affinity after three rounds from a promising population FH05. Different behavior of SELEX evolution could depend on the different character of the proteins.

Previously to these results, we had tried SELEX with nonmodified aptamers against the same proteins and we had not reached any positive result. The same behavior was observed by Gold et al. [23] in SELEX against a thousand of recombinant proteins from human serum, improving the positive results from $<30 \%$ with non-modified aptamers SELEX to $>90 \%$ with SOMAmers SELEX. Recently, some other groups have also improved the SELEX method with SOMAmers [33-35].

\subsection{Functionalization of the active surface of the resonators}

Surface functionalization of biosensors plays a critical role in the final performance of the devices. The most important issue is to cover the surface with a continuous and uniform layer of molecules avoiding the non-specific binding of undesirable molecules or reagents. We are looking for good reproducibility in the binding of aptamers to the surface and a background signal as low as possible.

It should be taken into account that the materials on the surface of the sensor in contact with the reagents are $\mathrm{SiO}_{2}$, on the active area, Mo, covering most of the surface, and AlN in a small amount around the active area. We have chosen Mo as the top surface layer 


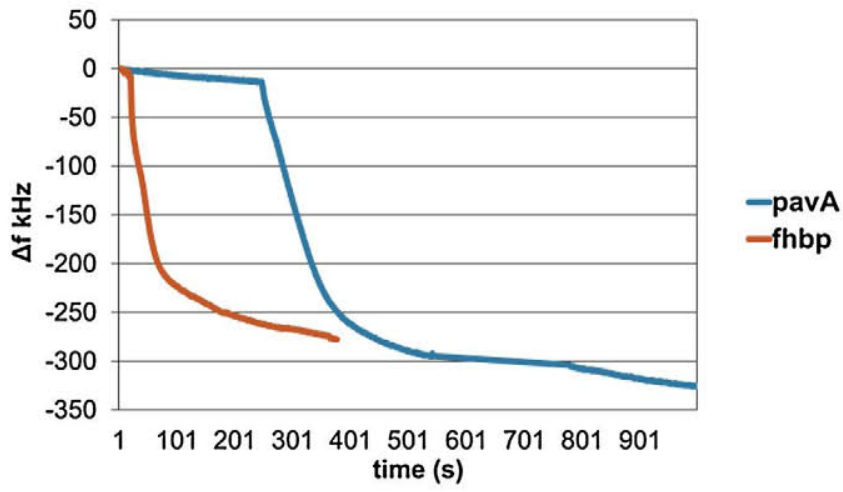

Fig. 2. Binding of PavA and FHbp His6-tag proteins were measured by the gravimetric sensor. Frequency shift indicates the binding of the proteins to the surface.

because Mo surface after $\mathrm{O}_{2}$ plasma treatment is not active and no APTES binds on it. After GA, the proteins were added at $500 \mathrm{nM}$ in $\mathrm{NaCl} 50 \mathrm{mM}$ solution recirculating with a peristaltic pump at $50 \mu \mathrm{l} / \mathrm{sec}$. Protein binding was measured by monitoring the resonant frequency of the gravimetric sensor with time. Fig. 2 shows the frequency shift as a function of time when PavA and FHbp proteins are bound to GA in an irreversible manner.

After protein binding, the surface was washed with SBIT and no frequency shift was detected in the gravimetric sensor, which ensures the anchoring of PavA and FHbp to the surface. A scheme of the functionalization and aptamer test is shown in Fig. 3.

Moreover, the presence of proteins on the surface was tested by adding a polyclonal antibody to His6-tag as binding positive control showing the corresponding frequency shift (data not shown). Non-specific binding was tested with some proteins, nucleic acids, surfactants and non-specific SOMAmers resulting in no modification of frequency (data not shown).

Frequency shift for PavA and FHbp binding is very similar for both proteins whereas PavA weights $64 \mathrm{kDa}$, almost double than FHbp $(30 \mathrm{kDa})$. This could be because the molecules have different sizes. The number of molecules of PavA necessary to cover the whole surface could be around half of FHbp resulting in the same total mass bound on the resonator surface as shown in Fig. 2, which shows the time evolution of the binding of both proteins. The similar steady state frequency shift obtained for both proteins indicates that the number of each of them per unit area are different. This would be probably due to the different sizes of molecules, being the number of reception sites occupied by the larger greater than those covered with the smaller.is necessary to use a functionalization method which avoids non-specific binding of any kind of

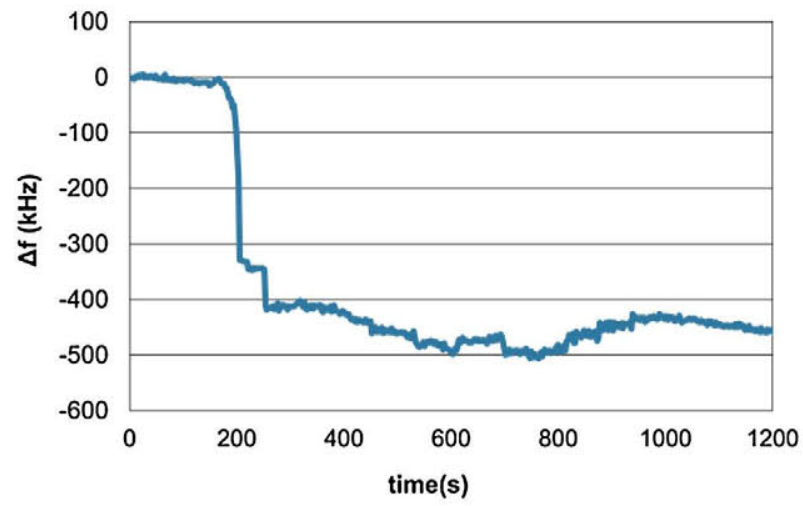

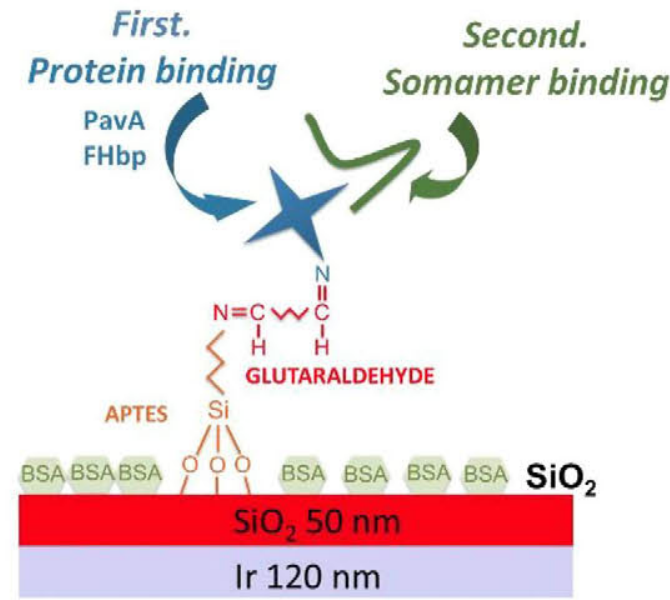

Fig. 3. The sensor active surface was functionalized with the proteins PavA or FHbp by the APTES-GA method. In a second step, the SOMAmer was incubated on the functionalized surface. During both processes, the mass bound to the surface was monitored.

molecules to the surface which could result in a frequency shift, namely, a false positive. In addition to this, the method of functionalization must keep the SOMAmers bonded after drying and rehydrating the aptasensor surface and the same surface has to avoid the non-specific binding as well. We performed different assays in this direction with very positive results for non-specific SOMAmers.

\subsection{Detection of SOMAmer binding to PavA and FHbp on resonator surface}

Once the proteins were immobilized on the resonator, the affinity of SOMAmer polyclonal populations FH05 and PA08 was tested. SOMAmers were added at $40 \mathrm{nM}$ in SBIT BSA $1 \%$, recirculating with a peristaltic pump at $50 \mu \mathrm{l} / \mathrm{sec}$. The binding reaction was measured in real time with the gravimetric sensor. Fig. 4 shows that the frequency shift in the case of PA08 is four times the frequency shift of FH05 and the slope indicates that the rate of reaction of PA08 is higher than the rate of reaction of FH05.

A greater frequency shift indicates more SOMAmers bound to the target as the molecular weight of both SOMAmers are the same. We attribute this to the PavA protein displaying in its structure more aptagenic epitopes than FHbp, resulting in more SOMAmers bound per PavA molecule. A faster rate of reaction indicates a polyclonal population with better affinity, surely because of the three

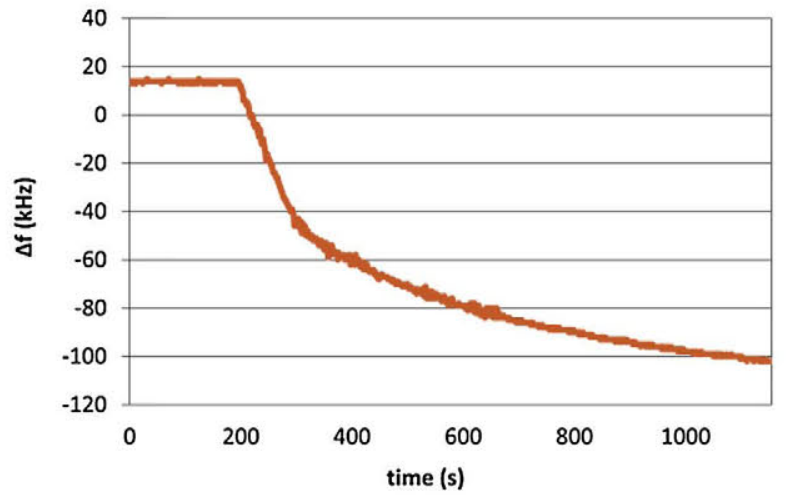

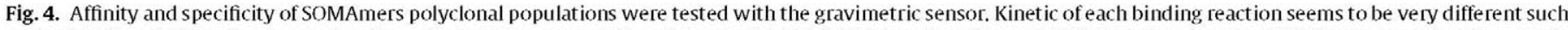

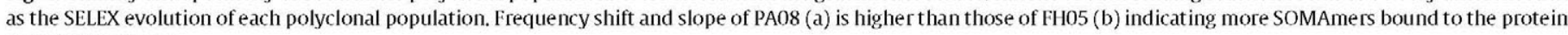
and higher affinity. 
more rounds of selection in PA08 than in FH05. Three more rounds from FH05 should achieve the same or similar affinity. In addition to the affinity test we incubated the PA08 and FH05 with different proteins coating the sensor surface with negative results, which demonstrate the specificity of such SOMAmers.

Further steps are needed for developing a successful aptasensor. Firstly, it is necessary to obtain the monoclonal SOMAmers by cloning and sequencing PA08 and FH05 polyclonal populations. After this, it is necessary to develop a functionalization protocol of the active surface of the sensors with those monoclonal SOMAmers showing the best affinity in order to test the aptasensor sensitivity.

According to the results obtained, the high frequency gravimetric aptasensor seems to be a promising biosensor to detect the specific proteins PavA and FHbp from N. meningitidis and S. pneumoniae surfaces. We have developed polyclonal populations of SOMAmers with high affinity for PavA and FHbp proteins and the functionalization method provides the aptasensor with SOMAmers bound to the surface in a stable manner. We have observed non measurable non-specific binding of other molecules, which is a very positive behavior. The reason for this could be due to the high speed of the surface movement in addition to the chemistry of functionalization, which impedes the permanence of non-well bound molecules on the surface. This phenomenon has to be carefully studied. Because of this exclusive feature of high frequency gravimetric sensors, they are very promising instruments for more applications, such as testing polyclonal and monoclonal SOMAmers and making SELEX directly to the target bound to the surface. All these new applications make the gravimetric high frequency sensor a promising tool with many more possibilities beyond the sensing.

\section{Conclusions}

SOMAmers against surface specific proteins PavA from Streptococcus pneumoniae and FHbp from Neisseria meningitidis have been selected and their affinity measured by direct ELONA and by gravimetric techniques using high frequency AlN resonators specially designed to operate in liquid. An APTES based functionalization protocol was used to fix the bacterium proteins to the surface of the sensors and the synthesized SOMAmers was detected in real time by binding them to the proteins. The SELEX method, the potential of the sensors and the affinity of SOMAmers to the proteins have been demonstrated being these the first steps towards the development of an aptasensor for Streptococcus pneumonia and Neisseria meningitides detection based on high frequency shear mode thin film resonators.

\section{Acknowledgments}

We want to thank Dr. Carlos Ferreirós for his advice and for providing bacteria strains of Neisseria meningitidis. Dr Sven Hammerschmidt for the advice with Streptococcus pneumoniae PavA protein.

This work was partially supported by the European Commission through the 7th Framework Programme by the RaptaDiag project HEALTH-304814, by COST action IC1208, and by Ministerio de Economía y Competitividad del Gobierno de España through the project MAT2013-45957-R.

\section{References}

[1] Meningococcal meningitis, Relevé Épidémiologique Hebd. Sect. Hygiène Secrétariat Société Nations Wkly. Epidemiol. Rec. Health Sect. Secr. Leag. Nations. 78 (2003) 294-296.

[2] U. Koedel, M. Klein, H.-W. Pfister, New understandings on the pathophysiology of bacterial meningitis, Curr. Opin. Infect. Dis. 23 (2010) 217-223, http://dx.doi,org/10.1097/QCO.0b013e328337f49e.
[3] C.L. Tacon, O. Flower, Diagnosis and management of bacterial meningitis in the paediatric population: a review, Emerg. Med. Int. 2012 (2012) 1-8, http:// dx.doi.org/10.1155/2012/320309.

[4] A.P. Cordeiro, R.A. Silva Pereira, A. Chapeaurouge, C.S. Coimbra, J. Perales, G. Oliveira, T.M. Sanchez Candiani, R.S. Coimbra, Comparative proteomics of cerebrospinal fluid reveals a predictive model for differential diagnosis of pneumococcal, meningococcal, and enteroviral meningitis, and novel putative therapeutic targets, BMC Genomics 16 (Suppl 5) (2015) S11, http:/) dx.doi.org/10.1186/1471-2164-16-s5-s11.

[5] A. Ahmed, J.V. Rushworth, N.A. Hirst, P.A. Millner, Biosensors for whole-cell bacterial detection, Clin. Microbiol. Rev. 27 (2014) 631-646, http://dx.doi.org/ 10.1128/CMR.00120-13.

[6] A.R. Tunkel, B.J. Hartman, S.L. Kaplan, B.A. Kaufman, K.L. Roos, W.M. Scheld, R.J. Whitley, Practice guidelines for the management of bacterial meningitis, Clin. Infect. Dis. 39 (2004) 1267-1284, http://dx.doi.org/10.1086/425368.

[7] M.J. Espy, J.R. Uhl, L.M. Sloan, S.P. Buckwalter, M.F. Jones, E.A. Vetter, J.D.C. Yao, N.L. Wengenack, J.E. Rosenblatt, F.R. Cockerill, T.F. Smith, Real-time PCR in clinical microbiology: applications for routine laboratory testing, Clin. Microbiol. Rev. 19 (2006) 165-256, http://dx.doi,org/10.1128/CMR.19.1.2006.

[8] J.V. Rushworth, N.A. Hirst, J.A. Goode, D.J. Pike, A. Ahmed, P.A. Millner, Impedimetric Biosensors for Medical Applications, ASME Press, 2013 (Accessed 9 June 2016) http://ebooks,asmedigitalcollection.asme.org/book. aspx?doi=10.1115/1.860243.

[9] C. Wu, Y. Zhou, X. Miao, L. Ling, A novel fluorescent biosensor for sequence-specific recognition of double-stranded DNA with the platform of graphene oxide, Analyst 136 (2011) 2106, http://dx.doi.org/10.1039/ c1an15061h.

[10] G. Kaur, A. Paliwal, M. Tomar, V. Gupta, Detection of Neisseria meningitidis using surface plasmon resonance based DNA biosensor, Biosens. Bioelectron. 78 (2016) 106-110, http://dx.doi.org/10.1016/j.bios.2015.11.025.

[11] D. Wang, G. Chen, H. Wang, W. Tang, W. Pan, N. Li, F. Liu, A reusable quartz crystal microbalance biosensor for highly specific detection of single-base DNA mutation, Biosens. Bioelectron. 48 (2013) 276-280, http://dx.doi.org/10. 1016/j.bios.2013.04,035.

[12] F. Lucarelli, S. Tombelli, M. Minunni, G. Marrazza, M. Mascini, Electrochemical and piezoelectric DNA biosensors for hybridisation detection, Anal. Chim. Acta 609 (2008) 139-159, http://dx.doi.org/10.1016/j.aca.2007.12.035.

[13] X. Tang, D. Zhao, J. He, F. Li, J. Peng, M. Zhang, Quenching of the electrochemiluminescence of tris(2,2'-bipyridine)ruthenium(II)/Tri- $n$ - propylamine by pristine carbon nanotube and its application to quantitative detection of DNA, Anal. Chem. 85 (2013) 1711-1718, http://dx.doi.org/10. $1021 / \mathrm{ac} 303025 \mathrm{y}$.

[14] A. Sotillo, M. Pedrero, M. de Pablos, J.L. García, E. García, P. García, J.M. Pingarrón, J. Mingorance, S. Campuzano, Clinical evaluation of a disposable amperometric magneto-genosensor for the detection and identification of Streptococcus pneumoniae, J. Microbiol. Methods 103 (2014) 25-28, http:// dx.doi.org/10.1016/j.mimet.2014.04.014.

[15] S.K. Dash, M. Sharma, A. Kumar, S. Khare, A. Kumar, Carbon composite-based DNA sensor for detection of bacterial meningit is caused by Neisseria meningitidis, J. Solid State Electrochem. 18 (2014) 2647-2659, http://dx.doi. org/10.1007/s10008-014-2525-9.

[16] S.K. Kailasa, H.-F. Wu, Nanomaterial-based miniaturized extraction and preconcentration techniques coupled to matrix-assisted laser desorption/ionization mass spectrometry for assaying biomolecules, TrAC Trends Anal. Chem. 65 (2015) 54-72, http://dx.doi.org/10.1016/j.trac.2014.09 011.

[17] S.K. Kailasa, H.-F. Wu, Surface modified BaTiO3 nanoparticles as the matrix for phospholipids and as extracting probes for LLME of hydrophobic proteins in Escherichia coli by MALDI-MS, Talanta 114 (2013) 283-290, http://dx.doi. org/10.1016/j.talanta.2013,05,032.

[18] S.K. Kailasa, H.-F. Wu, Dispersive liquid-liquid microextraction using functionalized $\mathrm{Mg}(\mathrm{OH}) 2$ NPs with oleic acid as hydrophobic affinity probes for the analysis of hydrophobic proteins in bacteria by MALDI MS, Analyst 137 (2012) 4490, http://dx.doi.org/10.1039/c2an35788g.

[19] S. Campuzano, B.E.-F. de Ávila, J. Yuste, M. Pedrero, J.L. García, P. García, E. García, J.M. Pingarrón, Disposable amperometric magnetoimmunosensors for the specific detection of Streptococcus pneumoniae, Biosens. Bioelectron. 26 (2010) 1225-1230, http://dx.doi.org/10.1016/j.bios.2010.06.011.

[20] S.B. Reddy, D.E. Mainwaring, M. Al Kobaisi, P. Zeephongsekul, J.V. Fecondo, Acoustic wave immunosensing of a meningococcal antigen using gold nanoparticle-enhanced mass sensitivity, Biosens. Bioelectron. 31 (2012) 382-387, http://dx.doi.org/10.1016/j.bios.2011.10.051.

[21] K.L. Hong, L.]. Sooter, Single-stranded DNA aptamers against pathogens and toxins: identification and biosensing applications, BioMed. Res. Int. 2015 (2015) 1-31, http://dx.doi.org/10.1155/2015/419318.

[22] J.D. Vaught, C. Bock, J. Carter, T. Fitzwater, M. Otis, D. Schneider, J. Rolando, S Waugh, S.K. Wilcox, B.E. Eaton, Expanding the chemistry of DNA for in vitro selection, J. Am. Chem. Soc. 132 (2010) 4141-4151, http://dx.doi.org/10.1021 ja908035g.

[23] L. Gold, D. Ayers, J. Bertino, C. Bock, A. Bock, E.N. Brody, J. Carter, A.B. Dalby, B.E. Eaton, T, Fitzwater, D. Flather, A. Forbes, T. Foreman, C. Fowler, B. Gawande, M. Goss, M. Gunn, S. Gupta, D. Halladay, J. Heil, J. Heilig, B. Hicke, G. Husar, N. Janjic, T. Jarvis, S. Jennings, E. Katilius, T.R. Keeney, N. Kim, T.H. Koch, S. Kraemer, L. Kroiss, N. Le, D. Levine, W. Lindsey, B. Lollo, W. Mayfield, M. Mehan, R. Mehler, S.K. Nelson, M. Nelson, D. Nieuwlandt, M. Nikrad, U. Ochsner, R.M. Ostroff, M. Otis, T. Parker, S. Pietrasiewicz, D.I. Resnicow, J. 
Rohloff, G. Sanders, S. Sattin, D. Schneider, B. Singer, M. Stanton, A. Sterkel, A. Stewart, S. Stratford, J.D. Vaught, M. Vrkljan, J.J. Walker, M. Watrobka, S. Waugh, A. Weiss, S.K. Wilcox, A. Wolfson, S.K. Wolk, C. Zhang, D. Zichi,

Aptamer-based multiplexed proteomic technology for biomarker discovery, PLoS One 5 (2010) e15004, http://dx.doi.org/10.1371/journal.pone.0015004.

[24] Chung-Jen Chung, Ying-Chung Chen, Chien-Chuan Cheng, Kuo-Sheng Kao, Synthesis and bulk acoustic wave properties on the dual mode frequency shift of solidly mounted resonators, IEEE Trans. Ultrason. Ferroelectr. Freq. Cont rol 55 (2008) 857-864, http://dx.doi.org/10.1109/TUFFC.2008.720.

[25] G. Sauerbrey, Verwendung von schwingquarzen zur Wägung dünner schichten und zur mikrowägung, Z. Für Phys. 155 (1959) 206-222, http://dx. doi.org/10.1007/BF01337937.

[26] J. Weber, W.M. Albers, J. Tuppurainen, M. Link, R. Gabl, W. Wersing, M. Schreiter, Shear mode FBARs as highly sensitive liquid biosensors, Sens. Actuators Phys. 128 (2006) 84-88, http://dx.doi.org/10.1016/j.sna.2006.01. 005 .

[27] G. Wingqvist, J. Bjurstrom, A. Hellgren, I. Katardjiev, Immunosensor utilizing a shear mode thin film bulk acoustic sensor, Sens. Actuators B Chem. 127 (2007) 248-252, http://dx.doi.org/10.1016/j.snb.2007.07.051.

[28] R. Ruby, A snapshot in time: the future in filters for cell phones, IEEE microw Mag. 16 (2015) 46-59, http://dx.doi.org/10.1109/MMM.2015,2429513.

[29] A.R. Holmes, R. McNab, K.W. Millsap, M. Rohde, S. Hammerschmidt, J.L Mawdsley, H.F. Jenkinson, The pavA gene of Streptococcus pneumoniae encodes a fibronectin-binding protein that is essential for virulence, Mol. Microbiol. 41 (2001) 1395-1408.

[30] M. Biagini, M. Spinsanti, G. De Angelis, S. Tomei, I. Ferlenghi, M. Scarselli, F. Rigat, N. Messuti, A. Biolchi, A. Muzzi, G. Anderloni, B. Brunelli, E. Cartocci, F. Buricchi, C. Tani, M. Stella, M. Moschioni, E. Del Tordello, A. Colaprico, S. Savino, M.M. Giuliani, I. Delany, M. Pizza, P. Costantino, N. Norais, R. Rappuoli, $\mathrm{V}$. Masignani, Expression of factor $\mathrm{H}$ binding protein in meningococcal strains can vary at least 15 -fold and is genetically determined, Proc. Natl. Acad. Sci. 113 (2016) 2714-2719, http://dx.doi.org/10.1073/pnas.152.1142113.

[31] M. DeMiguel-Ramos, T. Mirea, M. Clement, J. Olivares, J. Sangrador, E. Iborra, Optimized tilted c-axis AIN films for improved operation of shear mode resonators, Thin Solid Films 590 (2015) 219-223, http://dx.doi.org/10.1016/j. tsf.2015,08,010.

[32] M. DeMiguel-Ramos, B. Díaz-Durán, J.-M. Escolano, M. Barba, T. Mirea, J. Olivares, M. Clement, E. Iborra, Gravimetric biosensor based on a $1.3 \mathrm{GHz}$ AlN shear-mode solidly mounted resonator, Sens. Actuators B Chem. 239 (2017) 1282-1288, http://dx.doi.org/10.1016/j.snb.2016.09.079.

[33] T.C. Jarvis, D.R. Davies, A. Hisaminato, D.I. Resnicow, S. Gupta, S.M. Waugh, A Nagabukuro, T. Wadatsu, H. Hishigaki, B. Gawande, C. Zhang, S.K. Wolk, W.S. Mayfield, Y. Nakaishi, A.B. Burgin, L.J. Stewart, T.E. Edwards, A.D. Gelinas, D.J. Schneider, N. Janjic, Non-helical DNA triplex forms a unique aptamer scaffold for high affinity recognition of nerve growth factor, Structure 23 (2015) 1293-1304, http://dx.doi.org/10.1016/j.str.2015.03.027.

[34] A. Baumstummler, D. Lehmann, N. Janjic, U.A. Ochsner, Specific capture and detection of Staphylococcus aureus with high-affinity modified aptamers to cell surface components, Lett. Appl. Microbiol. 59 (2014) 422-431, http://dx. doi.org/10.1111/lam.12295.

[35] U.A. Ochsner, E. Katilius, N. Janjic, Detection of Clostridium difficile toxins A, B and binary toxin with slow off-rate modified aptamers, Diagn. Microbiol. Infect. Dis. 76 (2013) 278-285, http://dx,doi.org/10.1016/j.diagmicrobio,2013. 03.029 